\title{
Linear Cosmological Structure Limits on Warm Dark Matter
}

\author{
Kevork Abazajian \\ Theoretical Division, MS B285, Los Alamos National Laboratory, Los Alamos, NM 87545
}

\begin{abstract}
I consider constraints from observations on a cutoff scale in clustering due to free streaming of the dark matter in a warm dark matter cosmological model with a cosmological constant. The limits are derived in the framework of a sterile neutrino warm dark matter universe, but can be applied to gravitinos and other models with small scale suppression in the linear matter power spectrum. With freedom in all cosmological parameters including the free streaming scale of the sterile neutrino dark matter, limits are derived using observations of the fluctuations in the cosmic microwave background, the 3D clustering of galaxies and 1D clustering of gas in the Lyman-alpha (Ly $\alpha$ ) forest in the Sloan Digital Sky Survey (SDSS), as well as the Ly $\alpha$ forest in high-resolution spectroscopic observations. In the most conservative case, using only the SDSS main-galaxy 3D power-spectrum shape, the limit is $m_{s}>0.11 \mathrm{keV}$; including the SDSS Ly $\alpha$ forest, this limit improves to $m_{s}>1.7 \mathrm{keV}$. More stringent constraints may be placed from the inferred matter power spectrum from highresolution Ly $\alpha$ forest observations, which has significant systematic uncertainties; in this case, the limit improves to $m_{s}>3.0 \mathrm{keV}$ (all at $95 \% \mathrm{CL}$ ).

PACS numbers: 95.35.+d,14.60.Pq,14.60.St,98.65.-r
\end{abstract}

\section{INTRODUCTION}

The scale to which the ansatz of a (nearly) scaleinvariant power spectrum describes the cosmological primordial density fluctuations is unquantified at the smallest scales. The standard paradigm of an unmodified, nearly scale-invariant power spectrum arising from the amplification of quantum vacuum fluctuations of an inflaton field agrees well with the inferred matter power spectrum in the linear to mildly nonlinear regime of cosmological structure formation 1, 2, 3, 4, 5]. Models of the nonlinear clustering of galaxies are also consistent with a nearly scale-invariant perturbation spectrum [6].

There are a number of physical properties of the dark matter that may alter its perturbation spectrum at small scales. The hot big bang primordial plasma in the early universe may partially or completely thermodynamically couple to the dark matter. Any primordial velocity distribution that is imparted to the dark matter will allow dark matter to escape perturbations with sufficiently small gravitational potentials and damp structure formation at such scales. The most cited cold dark matter (CDM) candidate, the lightest supersymmetric particle (LSP), has a small but non-zero velocity dispersion, which damps structures below Earth mass scales [7, 8]. In warm dark matter (WDM) models, a light mass particle such as a sterile neutrino 9] or gravitino [10] with a large velocity dispersion can suppress linear structure formation up to galactic scales. The dark matter power spectrum may also be suppressed on small scales due to the production of the dark matter LSP through decay of charged progenitor causing a suppression of structure below the horizon scale at decay 11, 12 or inflation with broken scale invariance [13].

Models with a suppression of small scale power have drawn attention due to their potential alleviation of several unresolved problems in galaxy and small scale structure formation. These include, first, the reduction of satellite galaxy halos 14, 15, 16, 17], second, the reduction of galaxies in voids [18, 19], third, the low concentrations of dark matter in galaxies [20, 21, 22, 23], fourth, the angular momentum problem of galaxy formation 24], and fifth, the formation of disk-dominated galaxies [25, 26].

A significant reduction of power on sufficiently large scales will be in conflict with observations of clustering at small scales. It was noted by Narayanan et al. 27] that observations of power on the smallest linear scales in the Lyman-alpha (Ly $\alpha$ ) forest may provide particularly stringent constraints. In this paper, I examine the upper limits on the scale of such a suppression of small scale power from measures of matter clustering at small scales yet in the linear regime, where systematic effects in modeling can be minimized. I frame the constraints in terms of a sterile neutrino WDM candidate, motivated by new data and an accurate calculation of the transfer function for sterile neutrino dark matter based on an accurate calculation of the nonthermal production of sterile neutrino dark matter [28]. Limits on gravitino WDM and general departures of matter power spectra at small scales also can be inferred from these results. The smallscale clustering data employed here are the Sloan Digital Sky Survey (SDSS) main galaxy 3D power spectrum [29], the inferred linear matter power spectrum from observations of the flux power spectrum of Ly $\alpha$ absorption in the SDSS quasar catalogue [30, 31], and the inferred linear matter power spectrum from high-resolution observations of the Ly $\alpha$ forest of Croft et al. 32] as reanalyzed and augmented by Viel et al. [33] (hereafter VHS).

In order to alleviate degeneracies with other cosmolog- 
ical parameters, particularly the optical depth to the cosmic microwave background (CMB) and primordial scalar perturbation amplitude, as well as tighten constraints within allowable possibilities, I include observations of the fluctuations of the CMB from the first year Wilkinson Microwave Anisotropy Probe (WMAP) [34, 35], the Cosmic Background Imager (CBI) 36], Boomerang [37], the Arcminute Cosmology Bolometer Array (ACBAR) 38], and the Very Small Array (VSA) [39]. Viel et al. [40 have done a similar statistical analysis to that presented here, using WMAP and the inferred linear matter power spectrum of VHS to place constraints on a gravitino WDM candidate, which is applied to sterile neutrino dark matter via a central-value relationship. The work presented here is motivated by using a significantly more accurate transfer function for sterile neutrino dark matter of Ref. [28], as well as the results of inclusion of many more independent cosmological structure data.

It should be noted that observations of nonlinear structures may be a very powerful handle of primordial power at extremely small scales. The observations of anomalous flux ratios in gravitational lens systems can be an indication of substructure having a significant fraction of the mass of the lensing galaxies, which would not be present in certain WDM models [41, 42]. However, the exact level of the suppression of substructure that can be tolerated by these lensing observations is not clear, particularly given that some small mass halos may be formed by fragmentation in WDM models 43]; moreover, there may be a significant enhancement of the observed anomaly by line-of-sight isolated halos [4]. Another highly nonlinear process asserted to place strong constraints on the presence of small scale power is high-redshift (high- $z$ ) star formation inferred to be required to cause reionization early enough to produce the anomalously high $T E$ crosscorrelation at low multipoles seen by WMAP [45, 46]. CDM models themselves generally have difficulty in producing such high- $z$ reionization even when including the invocation of a very early generation of high-mass star formation [4], and it is not clear that the resolution of the problem of an anomalously high $T E$ cross-correlation at large scales observed by WMAP will be solved by the presence of small mass halos at high- $z$.

\section{ANALYSIS}

Here I present the results of an analysis of observational constraints on cosmological models with a cosmological constant and a general cold to warm dark matter in the form of a sterile neutrino. The standard cosmological model of structure formation from adiabatic Gaussian fluctuations seeded by an inflationary epoch is affected by perturbation growth in the radiation through matter dominated eras. The distribution of velocities of the dark matter suppresses fluctuations below its free streaming scale, which increases with the mean dark matter velocities and decreases with its mass.
The analysis here follows that of Refs. [2] and [3] , extended to include the possibility of a sterile neutrino warm dark matter candidate. The strong degeneracy that is present between the amplitude of the primordial scalar perturbations and the optical depth to the CMB [2] is exacerbated by the possibility of WDM. This results from the tightness of constraints on the matter fluctuation amplitude arising from the Ly $\alpha$ forest. Allowing a reduction of power at the smallest scales from WDM can be compensated by an increase in the primordial fluctuation amplitude, but made consistent with the $\mathrm{CMB}$ fluctuation amplitude by a high optical depth. There results a strong correlation between the sterile neutrino particle mass, the optical depth to the CMB, and the primordial fluctuation amplitude. This requires a prior to be set on the optical depth to the CMB in order to disallow unphysically high optical depths, $0.01<\tau<0.3$. All other parameters have flat priors well outside of nonzero likelihood values.

To constrain the cosmology in this analysis, I include the shape information from the SDSS 3D power spectrum of galaxies from the SDSS main galaxy sample 29], the McDonald et al. 31] inferred linear matter power spectrum from the analysis of the SDSS Ly $\alpha$ forest, as well as observations of the CMB by WMAP (first year), ACBAR $(\ell>800)$, CBI $(600<\ell<2000)$, VSA $(\ell>600)$, and BOOMERanG-2K2 34, 35, 36, 37, 38, 39, 48]. I also include a reanalysis by VHS of the linear matter power spectrum inferred from the high-resolution observations of the Ly $\alpha$ forest by the High Resolution Echelle Spectrometer of the Keck Observatory [32]. From VHS, I also include the inferred matter power spectrum from a new set of Ly $\alpha$ forest data obtained with the Ultraviolet and Visual Echelle Spectrograph (UVES) aboard the Very Large Telescope (VLT), called the Large sample of UVES QSO Absorption Spectra (LUQAS).

A useful form of the suppressed perturbation power spectrum for sterile neutrino dark matter, $P_{\text {sterile }}(k)$, relative to the CDM case is a transfer function of the form 28]

$$
T_{s}(k) \equiv \sqrt{\frac{P_{\text {sterile }}(k)}{P_{\mathrm{CDM}}(k)}},
$$

which can be used to convert any CDM transfer function to that of sterile neutrino dark matter. The fitting function that describes the transfer function is of the form

$$
T_{s}(k)=\left[1+(\alpha k)^{\nu}\right]^{-\mu},
$$

where

$$
\alpha=a\left(\frac{m_{s}}{1 \mathrm{keV}}\right)^{b}\left(\frac{\Omega_{\mathrm{DM}}}{0.26}\right)^{c}\left(\frac{h}{0.7}\right)^{d} h^{-1} \mathrm{Mpc},
$$

and $a=0.188, b=-0.858, c=-0.136, d=0.692$, $\nu=2.25$, and $\mu=3.08$. As the sterile neutrino particle mass decreases, the velocity distribution increases in 


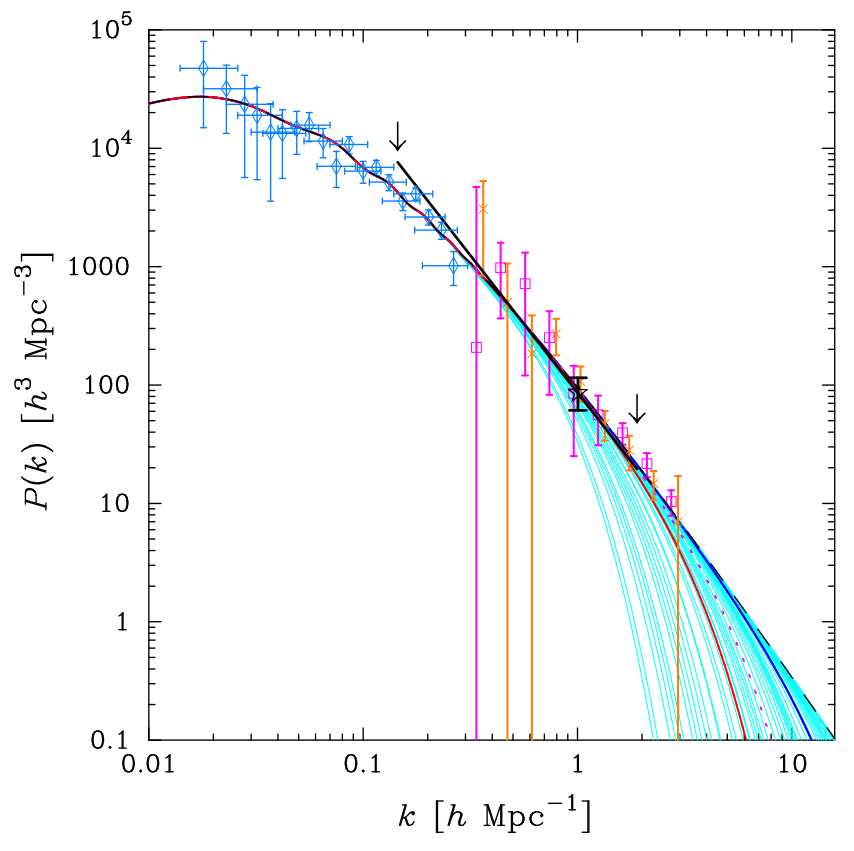

FIG. 1: Shown are the resulting linear matter power spectra $P(k)$ for a standard flat cosmological model $\Omega_{\mathrm{DM}}=0.26, \sigma_{8}=$ $0.9, \Omega_{b}=0.04$, and $h=0.7$ at $z=0$, and with sterile neutrino warm to cold dark matter in the mass range $0.3 \mathrm{keV}<m_{s}<$ $140 \mathrm{keV}$ (gray/cyan). The corresponding CDM case is dashed (black). Small-scale clustering data used here are the SDSS 3D power-spectrum of galaxies (diamonds), the inferred slope and amplitude of the matter power spectrum from SDSS Ly $\alpha$ forest observations (star point and slope between arrows), the inferred matter power spectrum from Ly $\alpha$ forest observations from Croft et al. [32] (cross points) and the LUQAS (square points), as interpreted by VHS [33]. Ly $\alpha$ forest measures are evolved to $z=0$ by the appropriate growth function. The solid (blue) line at high- $k$ is $P(k)$ for upper limit $m_{s}=8.2 \mathrm{keV}$ from observations of Virgo [49], the solid (red) line at low- $k$ is that for the lower limit from the SDSS Ly $\alpha$ forest in this work $\left(m_{s}=1.7 \mathrm{keV}\right)$, and the dotted line is that for the lower limit using high-resolution $\operatorname{Ly} \alpha$ forest data from this work $\left(m_{s}=3.0 \mathrm{keV}\right)$.

scale, and therefore the spatial scale $\alpha$ of the cutoff in the primordial clustering spectrum increases (see Fig. 1). The scale $\alpha$ represents where the power spectrum falls off by $11.8 \%$ relative to the standard CDM power spectrum, and can be used to place rough limits on other nonstandard dark matter models or broken scale-invariance inflation models 11, 12, 13]. The central values of the WDM sterile neutrino particle mass $m_{s}$ and thermal gravitino particle mass $m_{\tilde{g}}$ can be approximately related by [40]:

$$
m_{\tilde{g}} \approx 0.326 \mathrm{keV}\left(\frac{m_{s}}{1 \mathrm{keV}}\right)^{3 / 4}\left(\frac{\Omega_{\mathrm{DM}} h^{2}}{0.12}\right)^{1 / 4} .
$$

For a given cosmological parameter and sterile neutrino particle mass choice, we use the predicted matter power spectrum and CMB anisotropy angular correlation functions to calculate the likelihood to observe the combined data. The parameters we vary are the six parameters for the "vanilla" standard cosmological model plus the inverse sterile neutrino mass:

$$
\mathbf{p}=\left(\Omega_{b} h^{2}, \Omega_{d} h^{2}, \Theta_{s}, \ln (A), n, \tau, m_{s}^{-1}\right),
$$

where $\Omega_{b}$ and $\Omega_{d}$ are fractions of the critical density in baryons and dark matter; $\Theta_{s}$ is the angular acoustic peak scale of the CMB, a useful proxy for the Hubble parameter, $H_{0}=100 h \mathrm{~km} \mathrm{~s}^{-1} \mathrm{Mpc}^{-1} ; A$ and $n$ are the amplitude and tilt of the primordial scalar fluctuations; $\tau$ is the optical depth due to reionization. The inverse of $m_{s}$ is used to allow for a flat prior on and sampling of arbitrarily massive sterile neutrino models. To measure the likelihood space allowed by the data, we use a Markov Chain Monte Carlo (MCMC) method with a modified version of the Lewis and Bridle 50] CosmoMC code. We use the WMAP team's code to calculate the WMAP observations' likelihood, and CosmoMC to calculate that for Boomerang, ACBAR, CBI, and VSA. After burn-in, the chains typically sample $\gtrsim 10^{5}$ points, and convergence and likelihood statistics are calculated from these. Resulting marginalized 1D likelihoods for different data sets are shown in Fig. 2]

The shape of the 3D galaxy power spectrum from the main galaxy catalogue of the SDSS, $P_{g}(k)$ is a robust measure of the shape of the 3D matter power spectrum in the linear regime. The process of galaxy formation occurs on scales $\ll 10 \mathrm{Mpc}$ 51], where gas cools within dark matter halos and the large scale clustering then follows that of the total matter components [52]. Therefore, using the shape information from the 3D power spectrum of SDSS galaxies at $k<0.2 h^{-1} \mathrm{Mpc}$ is a very conservative upper bound on the deviations of the primordial spectrum due to WDM. The lower limit on a sterile neutrino dark matter candidate in this case is $m_{s}>0.108 \mathrm{keV}$, gravitino WDM particle mass $m_{\tilde{g}}>0.063 \mathrm{keV}$ and cutoff scale $\alpha<1.22 h^{-1} \mathrm{Mpc}$, at 95\% CL, with 1D likelihoods shown in Fig. [3]

Observations of spectra of distant quasars contain absorption features from the Ly $\alpha$ transition due to neutral hydrogen gas along the line of site. The numerous absorption features are a Ly $\alpha$ "forest" that follows gas in-fall into gravitational potentials along the line of site and therefore can be used to infer the power spectrum of dark matter density along this line of site. The statistic chosen to parametrize the clustering of the Ly $\alpha$ features is the power spectrum of the transmitted flux fraction $P_{F}(k, z)$. The relation between the gas and dark matter density relies on the state of ionization equilibrium of the gas, and in turn is dependent on the temperature-density relation, the intensity of the UV background, the mean baryon density and many other physical parameters.

With tests via hydrodynamic simulations, one approach is mapping the relation between the $1 \mathrm{D} P_{F}(k, z)$ and 3D matter $P(k, z)$, which then can be inverted through a biasing relation $P_{F}(k, z)=b^{2}(k, z) P(k, z)$. This is the approach of VHS, is what is used by Viel et al. 40 to place a limit on the WDM free streaming scale and lower limit on the WDM particle mass, and is 

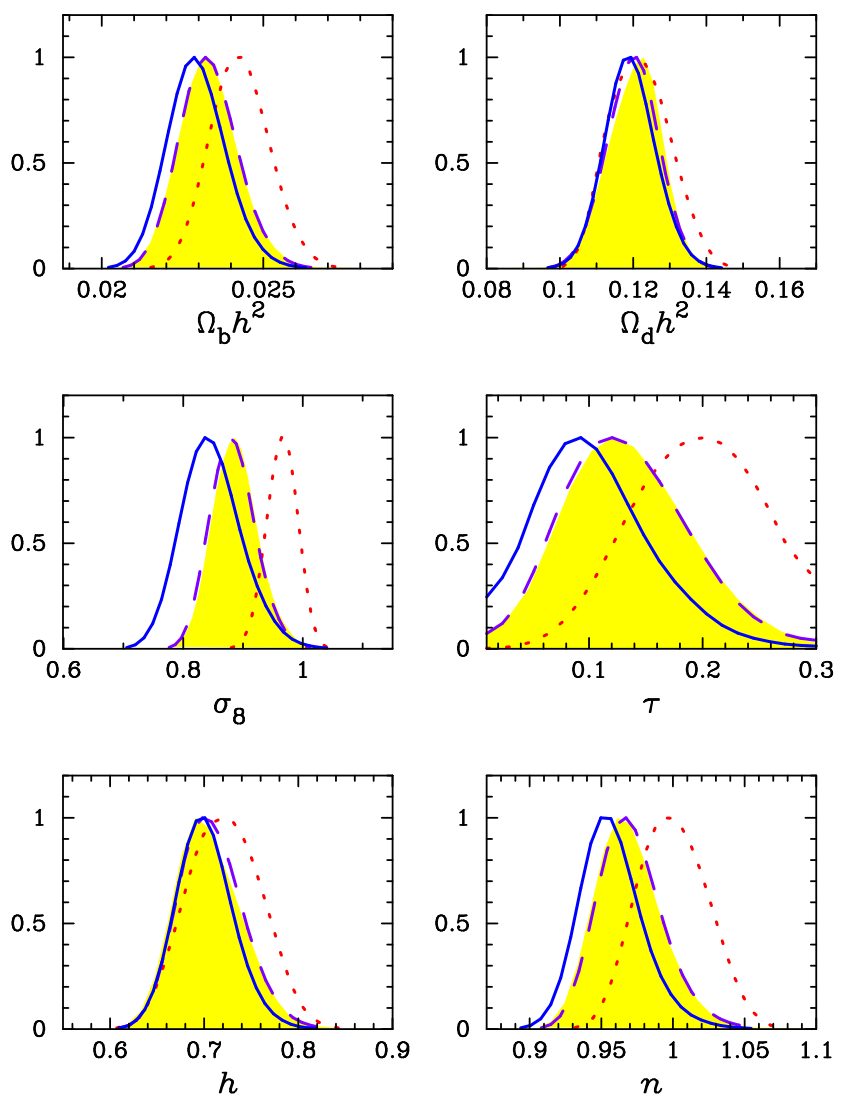

FIG. 2: Shown here are the 1D marginalized likelihoods on chosen base and derived cosmological parameters for the CMB plus SDSS 3D $P_{g}(k)$ in solid (blue), plus SDSS Ly $\alpha$ forest in dashed (purple), plus the VHS high-resolution Ly $\alpha$ forest in dotted (red). The shaded (yellow) likelihoods are for the CMB plus SDSS 3D $P_{g}(k)$ plus SDSS Ly $\alpha$ forest for the standard CDM case, which shows that parameters are generally not biased when including the presence of WDM.

my method of applying their results here. A key result of Ref. [40] is that $b^{2}(k, z)$ is nearly identical for CDM and WDM over the wave-numbers used in the inversion of $P_{F}(k, z)$. This motivates applying the results of CDMbased analyses to a WDM model, though the differences between WDM and CDM models in power spectrum inversion should be explored further. It is important to note that there is much contention regarding the accuracy of the inversion $b^{2}(k, z)$ (see, e.g., Refs. [53, 54, 55]), especially regarding mapping the full parameter space of the inversion $b^{2}(k, \mathbf{p}, \mathbf{q})$ in cosmological $\mathbf{p}$ and physical parameters $\mathbf{q}$ of the gas.

Instead of a direct relation between the flux and matter power spectra, McDonald et al. 31 map out the likelihood space for the amplitude and slope of the linear matter power spectrum for the SDSS Ly $\alpha$ forest data for a given range of physical parameters, in order to quantify much of the systematic effects of the uncertain physical states of the gas in the modeling. Note that Viel et al. 56 have done a reanalysis of the inferred matter power spectrum using a very different analysis and get
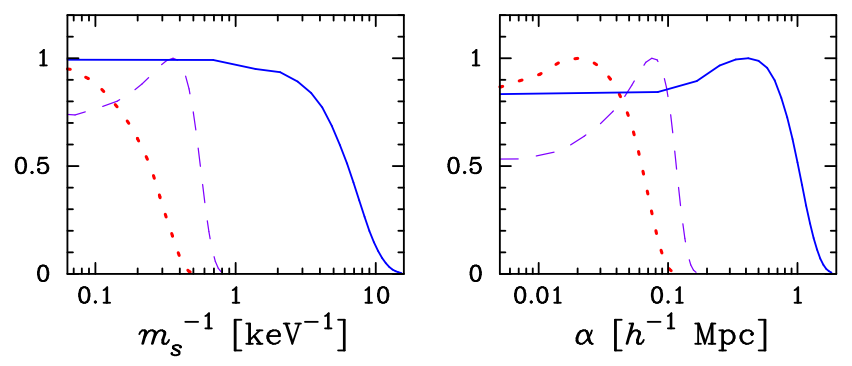

FIG. 3: Shown here are the 1D marginalized likelihoods for the (inverse) mass of the sterile neutrino dark matter, $m_{s}$ and the scale of the primordial spectrum cutoff $\alpha$, from the CMB plus SDSS 3D $P_{g}(k)$ in solid (blue), plus SDSS Ly $\alpha$ forest in dashed (purple), and plus the VHS high-resolution Ly $\alpha$ forest in dotted (red).

consistent results with McDonald et al. Since the systematic effects are generally non-Gaussian in likelihood space, McDonald et al. therefore provide a likelihood look-up table for the amplitude and slope of the matter power spectrum at the effective redshift of the SDSS Ly $\alpha$ forest. Using this likelihood from this interpretation of the SDSS Ly $\alpha$ forest data, combined with the base model of the CMB plus SDSS 3D $P_{g}(k)$, provides an order of magnitude more stringent constraint on the free streaming scale and WDM particle mass: $m_{s}>1.71 \mathrm{keV}$, $m_{\tilde{g}}>0.500 \mathrm{keV}$ and $\alpha<0.121 h^{-1} \mathrm{Mpc}$, at $95 \% \mathrm{CL}$. It should be noted that the $1 \mathrm{D}$ likelihood for the cutoff scale and inverse sterile neutrino mass peak at non-vanishing values (Fig. 3), and indicate the possibility of detecting a small scale cutoff through Ly $\alpha$ forest observations.

The high-resolution Ly $\alpha$ forest data analyzed by VHS can provide an even more stringent constraint on the free streaming scale of the WDM and particle mass, and I include that data as done in Ref. [40]. I use the inferred matter power spectrum of VHS, Table 4, but reduced by a uniform $7 \%$ for a change in the temperature-density relation, as done in Refs. 4, 40]. In addition, there are systematic uncertainties as described in Refs. [4, 40], namely for the power and amplitude of the temperature density relation $(5 \%)$, the measure of the effective optical depth for calibration $(8 \%)$, the uncertainty in the $b^{2}(k, z)$ inversion $(5 \%)$, uncertainty in the effects of galactic winds $(5 \%)$, and finally an uncertainty due to differences in numerical simulations (8\%). These systematic errors are added in quadrature to give an overall systematic uncertainty of $14.5 \%$, which is comparable to the statistical uncertainties to which it is, in turn, added in quadrature. It is certainly unknown how correct the assumption is that these systematic errors are Gaussian and should be combined in quadrature. However, a more robust analysis of the high-resolution Ly $\alpha$ forest data does not yet exist, and so I follow the procedure of using the VHS data as in Refs. [4, 40], with the above caveats in mind. Using the VHS high-resolution data in concert with the SDSS 3D $P_{g}(k)$ and SDSS Ly $\alpha$ forest gives the constraints: $m_{s}>3.00 \mathrm{keV}, m_{\tilde{g}}>0.769 \mathrm{keV}$ and 


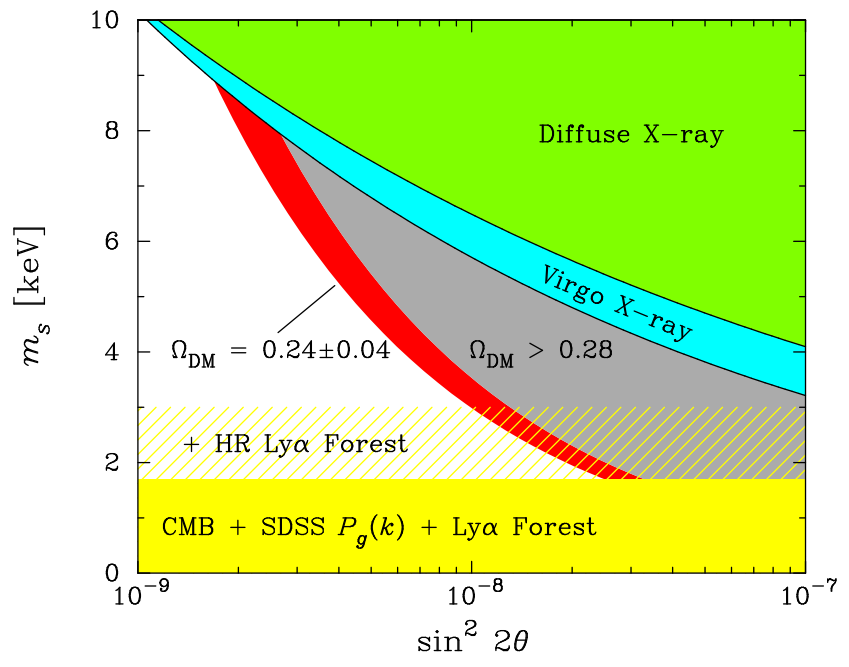

FIG. 4: The parameter space for sterile neutrino dark matter production and constraints. The red (dark grey) band is consistent with sterile neutrinos composing the dark matter consistent with $\Omega_{\mathrm{DM}}=0.24 \pm 0.04$ [28]. Upper limits arise from radiative decay limits from XMM-Newton X-ray observations of the Virgo Cluster [4] and the diffuse X-ray background 57]. The lower limits of this work from the CMB plus SDSS 3D $P_{g}(k)$ plus SDSS Ly $\alpha$ are in solid (yellow) at $m_{s}<1.7 \mathrm{keV}$ and all previous data plus VHS in dashed (yellow) at $m_{s}<3.0 \mathrm{keV}$.

$\alpha<0.0756 h^{-1} \mathrm{Mpc}$, at $95 \% \mathrm{CL}$. A summary of the parameter space for sterile neutrino dark matter is shown in Fig. 4]

\section{CONCLUSIONS}

Potential problems in galaxy and small-scale structure formation indicate the possibility of a small-scale velocity damping of perturbations of the type in warm dark matter such a sterile neutrino dark matter. This work presents a calculation of cosmological parameter constraints including the possibility of a warm dark matter candidate to investigate the largest scale of the suppression of small scale power allowed by linear measures of the cosmological structure from the inferred matter power spectrum. I find the lower limits on a sterile neutrino dark matter particle mass placed by observations of fluctuations in the cosmic microwave background, clustering of galaxies and in the Ly $\alpha$ forest in the Sloan Digital Sky Survey (SDSS), and clustering of the Ly $\alpha$ forest in high-resolution spectroscopic observations by the Keck and VLT Observatories. The lower mass bound in the most conservative case, using only the SDSS maingalaxy 3D power-spectrum shape is $m_{s}>0.11 \mathrm{keV}$; including the SDSS Ly $\alpha$ forest, this limit improves to $m_{s}>1.7 \mathrm{keV}$, while also including high-resolution Ly $\alpha$ forest data, this improves to $m_{s}>3.0 \mathrm{keV}$, with all lim- its at $95 \%$ CL. These results are consistent with the results of Viel et al. [40], but more constrained than their results $\left(m_{s}>2.0 \mathrm{keV}\right)$ due to the inclusion here of additional CMB and large scale structure data. The limits from the high-resolution Ly $\alpha$ forest data are subject to large systematic uncertainties, and therefore I adopt the SDSS Ly $\alpha$ data as the current WDM structure benchmark. These constraints can also be inferred to place limits on gravitino warm dark matter candidates as well as other models with a primordial fluctuation small scale cutoff, $\alpha$. The central values and confidence intervals for the other cosmological parameters do not change significantly with the inclusion of the possibility of WDM.

The sterile neutrino WDM candidate is well constrained by the linear clustering observations considered here, and by limits on its radiative decay by observations of the Virgo cluster by XMM-Newton, which provides an upper mass constraint [49, [58]. There also exist upper mass constraints from the diffuse X-ray background [57, 59]. The combination of these constraints allows a narrow range for this particle dark matter candidate mass:

$$
1.7 \mathrm{keV}<m_{s}<8.2 \mathrm{keV} \text {. }
$$

It remains for further work to detect or constrain X-ray lines from nearby clusters of galaxies, as well as use the presence or lack of a cutoff scale in the linear matter power spectrum to detect or exclude this dark matter candidate.

\section{Acknowledgments}

It is a pleasure to thank Neal Dalal, Salman Habib, Katrin Heitmann, Julien Lesgourgues and Matteo Viel for useful discussions. This work was supported by Los Alamos National Laboratory under DOE contract W7405-ENG-36.

Note added in proof: A recent preprint, Seljak et al. astro-ph/0602430 has suggested significantly more stringent constraints on the free streaming scale of the WDM than that presented here $\left(m_{s}>14 \mathrm{keV}, 95 \% \mathrm{CL}\right)$. The essential difference in that work is claimed to be the higher spatial resolution in their numerical simulations which resolve the high sensitivity of the $1 \mathrm{D}$ flux power spectrum to a reduction in small-scale power relative to the 3D power spectrum. However, the spatial resolution of the grid in simulations in Narayanan et al [27] is higher $\left(49 h^{-1} \mathrm{kpc}\right)$ than that in astro-ph/0602430 $\left(78 h^{-1} \mathrm{kpc}\right)$, yet Narayanan et al. see essentially no difference in highresolution flux power spectra between CDM and $1 \mathrm{keV}$ thermal WDM (4.4 keV sterile neutrinos). Therefore, it is not clear from where the claimed stringent constraints in astro-ph/0602430 arise. 
[1] D. N. Spergel et al. (WMAP), Astrophys. J. Suppl. 148, 175 (2003), astro-ph/0302209.

[2] M. Tegmark et al. (SDSS), Phys. Rev. D69, 103501 (2004), astro-ph/0310723.

[3] U. Seljak et al., Phys. Rev. D71, 103515 (2005), astro$\mathrm{ph} / 0407372$.

[4] M. Viel, J. Weller, and M. Haehnelt, Mon. Not. Roy. Astron. Soc. 355, L23 (2004), astro-ph/0407294.

[5] K. Abazajian, K. Kadota, and E. D. Stewart, JCAP 0508, 008 (2005), astro-ph/0507224.

[6] K. Abazajian et al., Astrophys. J. 625, 613 (2005), astro$\mathrm{ph} / 0408003$.

[7] S. Hofmann, D. J. Schwarz, and H. Stoecker, Phys. Rev. D64, 083507 (2001), astro-ph/0104173.

[8] A. M. Green, S. Hofmann, and D. J. Schwarz, JCAP 0508, 003 (2005), astro-ph/0503387.

[9] S. Dodelson and L. M. Widrow, Phys. Rev. Lett. 72, 17 (1994), hep-ph/9303287.

[10] G. R. Blumenthal, H. Pagels, and J. R. Primack, Nature 299, 37 (1982).

[11] K. Sigurdson and M. Kamionkowski, Phys. Rev. Lett. 92, 171302 (2004), astro-ph/0311486.

[12] M. Kaplinghat, Phys. Rev. D72, 063510 (2005), astro$\mathrm{ph} / 0507300$.

[13] M. Kamionkowski and A. R. Liddle, Phys. Rev. Lett. 84, 4525 (2000), astro-ph/9911103.

[14] G. Kauffmann, S. D. M. White, and B. Guiderdoni, Mon. Not. Roy. Astron. Soc. 264, 201 (1993).

[15] A. A. Klypin, A. V. Kravtsov, O. Valenzuela, and F. Prada, Astrophys. J. 522, 82 (1999), astro$\mathrm{ph} / 9901240$.

[16] B. Moore et al., Astrophys. J. 524, L19 (1999), astro$\mathrm{ph} / 9907411$.

[17] B. Willman, F. Governato, J. Wadsley, and T. Quinn, Mon. Not. Roy. Astron. Soc. 353, 639 (2004), astro$\mathrm{ph} / 0403001$.

[18] P. J. E. Peebles (2001), astro-ph/0101127.

[19] P. Bode, J. P. Ostriker, and N. Turok, Astrophys. J. 556, 93 (2001), astro-ph/0010389.

[20] J. J. Dalcanton and C. J. Hogan, Astrophys. J. 561, 35 (2001), astro-ph/0004381.

[21] F. C. van den Bosch and R. A. Swaters, Mon. Not. Roy. Astron. Soc. 325, 1017 (2001), astro-ph/0006048.

[22] A. R. Zentner and J. S. Bullock, Phys. Rev. D66, 043003 (2002), astro-ph/0205216.

[23] K. Abazajian, S. M. Koushiappas, and A. R. Zentner, in preparation (2005).

[24] A. D. Dolgov and J. Sommer-Larsen, Astrophys. J. 551, 608 (2001).

[25] F. Governato et al., Astrophys. J. 607, 688 (2004), astro$\mathrm{ph} / 0207044$.

[26] J. Kormendy and D. B. Fisher, Revista Mexicana de Astronomia y Astrofisica (Conference Series) 25, 101 (2005), astro-ph/0507525.

[27] V. K. Narayanan, D. N. Spergel, R. Dave, and C.-P. Ma, Astrophys. J. 543, L103 (2000), astro-ph/0005095.

[28] K. Abazajian, [Phys. Rev. D (to be published)] (2005), astro-ph/0511630.

[29] M. Tegmark et al. (SDSS), Astrophys. J. 606, 702 (2004), astro-ph/0310725.

[30] P. McDonald et al. (2004), astro-ph/0405013.
[31] P. McDonald, U. Seljak, R. Cen, D. Shih, D. H. Weinberg, S. Burles, D. P. Schneider, D. J. Schlegel, N. A. Bahcall, J. W. Briggs, et al., Astrophys. J. 635, 761 (2005).

[32] R. A. C. Croft et al., Astrophys. J. 581, 20 (2002), astro$\mathrm{ph} / 0012324$.

[33] M. Viel, M. G. Haehnelt, and V. Springel, Mon. Not. Roy. Astron. Soc. 354, 684 (2004), astro-ph/0404600.

[34] G. Hinshaw et al., Astrophys. J. Supp. 148, 135 (2003), astro-ph/0302217.

[35] A. Kogut et al., Astrophys. J. Supp. 148, 161 (2003), astro-ph/0302213.

[36] A. C. S. Readhead et al., Astrophys. J. 609, 498 (2004), astro-ph/0402359.

[37] W. C. Jones et al. (2005), astro-ph/0507494.

[38] C.-l. Kuo et al. (ACBAR), Astrophys. J. 600, 32 (2004), astro-ph/0212289.

[39] C. Dickinson et al., Mon. Not. Roy. Astron. Soc. 353, 732 (2004), astro-ph/0402498.

[40] M. Viel, J. Lesgourgues, M. G. Haehnelt, S. Matarrese, and A. Riotto, Phys. Rev. D71, 063534 (2005), astro$\mathrm{ph} / 0501562$.

[41] N. Dalal and C. S. Kochanek, Astrophys. J. 572, 25 (2002), astro-ph/0111456.

[42] C. S. Kochanek and N. Dalal, Astrophys. J. 610, 69 (2004), astro-ph/0302036.

[43] A. Knebe, J. E. G. Devriendt, B. K. Gibson, and J. Silk, Mon. Not. Roy. Astron. Soc. 345, 1285 (2003), astro$\mathrm{ph} / 0302443$.

[44] J. Chen, A. V. Kravtsov, and C. R. Keeton, Astrophys. J. 592, 24 (2003), astro-ph/0302005.

[45] R. Barkana, Z. Haiman, and J. P. Ostriker, Astrophys. J. 558, 482 (2001), astro-ph/0102304.

[46] N. Yoshida, A. Sokasian, L. Hernquist, and V. Springel, Astrophys. J. 591, L1 (2003), astro-ph/0303622.

[47] J. M. Rozas, J. Miralda-Escude, and E. Salvador-Sole (2005), astro-ph/0511489.

[48] L. Verde et al., Astrophys. J. Supp. 148, 195 (2003), astro-ph/0302218.

[49] K. Abazajian, G. M. Fuller, and W. H. Tucker, Astrophys. J. 562, 593 (2001), astro-ph/0106002.

[50] A. Lewis and S. Bridle, Phys. Rev. D66, 103511 (2002), astro-ph/0205436.

[51] S. D. M. White and M. J. Rees, Mon. Not. Roy. Astron. Soc. 183, 341 (1978).

[52] D. H. Weinberg, R. Dave, N. Katz, and L. Hernquist, Astrophys. J. 601, 1 (2004), astro-ph/0212356.

[53] M. Zaldarriaga, R. Scoccimarro, and L. Hui, Astrophys. J. 590, 1 (2003), astro-ph/0111230.

[54] U. Seljak, P. McDonald, and A. Makarov, Mon. Not. Roy. Astron. Soc. 342, L79 (2003), astro-ph/0302571.

[55] T. Jena et al., Mon. Not. Roy. Astron. Soc. 361, 70 (2005), astro-ph/0412557.

[56] M. Viel and M. G. Haehnelt, Mon. Not. Roy. Astron. Soc. 365, 231 (2006), astro-ph/0508177.

[57] A. Boyarsky, A. Neronov, O. Ruchayskiy, and M. Shaposhnikov (2005), astro-ph/0512509.

[58] H. Böhringer et al., Astron. \& Astrophys. 365, L181 (2001), astro-ph/0011459.

[59] M. Mapelli and A. Ferrara (2005), astro-ph/0508413. 